\title{
Multi-sensor System for Level Measurements with Optical Fibres
}

\author{
C. Vázquez, J. Garcinuño, J. M. S. Pena, and A. B. Gonzalo
}

Dpto. Tecnología Electrónica, Escuela Politécnica Superior

Universidad Carlos III Madrid,

Avenida Universidad 30

28911 Leganés, Madrid, SPAIN

cvazquez@ing.uc3m.es

\begin{abstract}
A system for measuring liquid level in multiple tanks using optical fibre technology has been developed. Oil field service industry can be benefited from this intrinsically safe technology. Plastic optical fibre (POF) sensor heads are excited by a $650 \mathrm{~nm}$ laser. Laser diodes are housed in ST connectors to obtain compact and rough prototypes and these connectors are also used in the fibre pigtails. Optical multiplexing is used to increase the measuring safety area. POF splitters and connectors are used to combine all the receiving sensor head fibres in a single one. Frequency Division Multiplexing is used to address each sensor head. The global system is controlled through a user friendly software application running in a PC connected to the system via an RS-232 port. A scalable prototype with a range greater than 2 meter, good linearity, better than $1.5 \%$ FS (full scale), high accuracy and resolution is developed using a unique lens to collimate and focus the light. Measurements are taken to validate the designs. Up to 8 sensor heads can be connected in the present implementation. But a greater number of sensors can be allocated with minor modifications in the electronics.
\end{abstract}

\section{INTRODUCTION}

Intrinsically safe measurements are critical in a wide range of industrial environments such as inflammable atmospheres. Nowadays, a visual inspection of fuel level in open tanks is carried out in many petrol stations. In this way, the need to fill up is detected along with a control of fuel delivery. But, Volatile Organic Compounds (VOC) are emitted while opening the tanks, deteriorating ozone layer and being able to hurt operator health. The VOC's emission was the $20 \%$ of the total pollutant emission in 1998 [1]. Although the inspection process is relatively fast, this process is carried out many times in many petrol stations. Electronic systems have been developed and financed for installation in countries such as Mexico [2] to overcome the above mentioned problems.

There are several methods for measuring and remote monitoring liquid levels. Mechanical float-type level indicating devices have been used to measure fluid levels by placing a float in the vessel itself. This method provides accurate readings but requires frequent calibration and maintenance because it is an intrusive method so the liquid can deteriorate the hardware. Other disadvantage is that the electronics must be kept close to the measuring point. Ultrasonic level detectors avoid direct contact with the fluid, using a transmitter/receiver but suffers from inaccuracies causes by varying temperatures and densities in the area above the fluid level and from potential confusion of echoes [3]. Microwave and radar indicators also avoid contact with the fluid but in some designs inaccurate measurements are obtained in fluids with poor dielectric constants. Depending on the operation frequency, the antenna dimensions can limit the minimal distance to the liquid surface to be measured. Even more, all previous methods are electronic in nature so they suffer from intrinsic safety concerns. As with any electronic device, there are necessarily, heat generating components that create, no matter how remote, the possibility of hazardous situations when used in areas where flammable materials are present. So an extra cost must be considered to make these techniques suitable for inflammable atmospheres with no risk.

Optical technologies with optical fibres are intrinsically safe in nature, with no risk of explosion even under malfunction operation, because inside the tank and the surroundings there is only inert materials such as optical fibres. The optical technology has other advantages such as no EMI, low weight and as a transmission media: low loss and wide bandwidth.

Different laser and optical instrumentation devices have been already used in level measurement systems, such as the patent described in [4]. But in any of them the laser, so the electronic driver, is in the sensor head. To avoid electronic presence in the measuring point, optical fibres should be used in the sensor head. This idea has been used in different optical sensors but for measuring short distances or just as simple control level devices [5-8]. A remote sensor head based on optical fibres for long distance is reported in [9] but using different lenses from transmitter and receiver fibres with the complexion related to this disposition, the measuring principle is different and none optical multiplexing is discussed.

In this paper, a multi-sensor system for level measurements which is based on optical fibres to be used in the head transducers and as the transmission media along the optical network, is presented. Multiple tanks or point measurements are addressed using Frequency Division Multiplexing and optical fibre directional couplers. Optoelectronics circuits are developed for properly conditioning the laser output and the light reflected off the liquid surface. Control and Data acquisition in the system is developed using a microcontroller which is connected via RS-232 port to the PC. Novel principle of operation is also discussed and 2 different sensor heads are characterized.

\section{STRUCTURE, DESIGN AND DEVELOPMENT}

A schematic of the multi-sensor, scalable system can be 


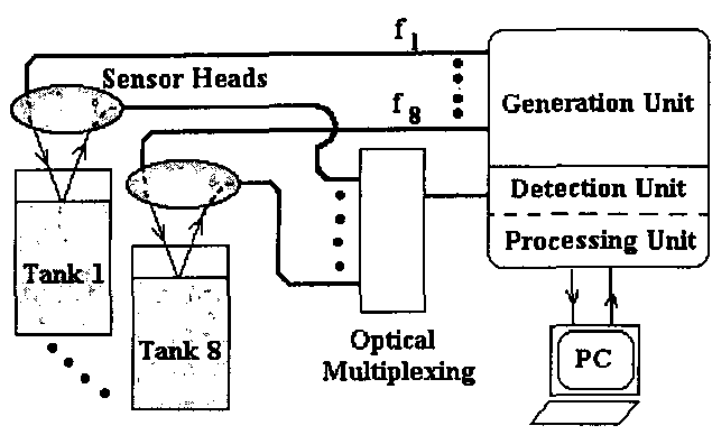

Fig. 1. A schematic of the multi-sensor system

seen in Fig. 1. Optical fibres are used in the sensor heads and for optical multiplexing to address different tanks without using multiple cables. This system allows an intrinsically safe level measurement with a simple, modular and cost effective solution. In the following we are going to describe the general architecture, the measuring principle and a more detailed description of the different blocks. Design and development aspects will be covered.

\section{A. Principle of operation}

The attenuation of the light transmitted from the sensor, reflected off the liquid surface and returned to the receiver fibre depends on the distance from the sensor head to the liquid surface.

A unique lens is used to collimate the incident beam and to focus the reflected beam. The emitter fibre is placed in the lens' focal plane, near the focus, $f$. Therefore, behind the lens, there is a collimated beam that is tilted a little angle, referred to the lens optical axis. The beam is reflected by the liquid surface and come back to the lens (see discontinuous trace on Fig. 2). Due to the mentioned angle, the beam suffers a lateral displacement, that depends on the distance between the lens and the liquid surface, $D$. Consequently, the lens collects only a part of the beam, whose image is formed on the focal plane, in a point symmetric to the emitter fibre in relation to the focus. The receptor fibre is placed in this position.

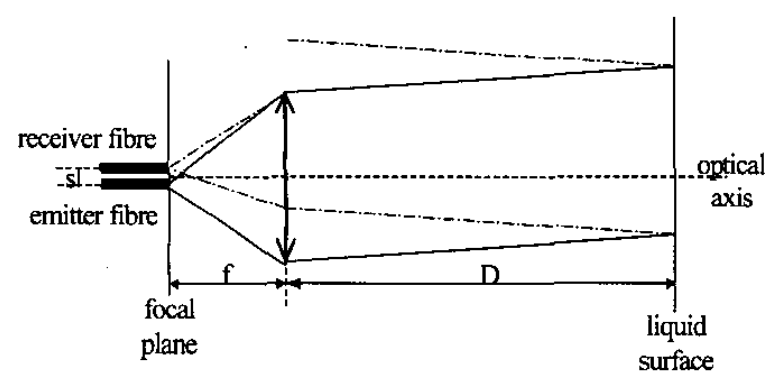

Fig. 2. A schematic showing the incident ___and reflected --- beam path depending on the location of the lens and the emitter and receiver fibres.
Assuming that:

- the absorption of the laser radiation by the air is negligible,

- the emitter fibre is placed in a way that fibre numerical aperture does not produce any limitation,

- the power is uniformly distributed in the laser cross section;

the signal generated by the photodiode, which is placed behind the receptor fibre should be proportional to the rate of the common area between the reflected beam and the lens, $S_{\text {com }}$, to the total area of the reflected beam, $S_{\text {tot. }}$ As the emitter fibre is very close to the lens focus, $S_{\text {com }}$ could be calculated as the common area of two intersecting circumferences (see Fig. 3).

Being $a_{I}$ the lens radius, $a_{2}$ the reflected beam radius, $\mathrm{s}$ the distance between the fibre centres and $D$ the distance between the lens and the liquid surface, the expression for the photodiode signal , $P$, is as follows:

$P=k \frac{a_{1}^{2}\left(Z_{1}-\sin Z_{1}\right)+a_{2}^{2}\left(Z_{2}-\sin Z_{2}\right)}{2 \pi a_{2}^{2}}$,

where $Z_{1}$ and $Z_{2}$ are functions of $a_{1}, a_{2}$ and $c$. Being $c$ the distance between the centres of the circumferences shown in Fig. 3, which is given by:

$c=s \frac{D}{f}$,

$k$ is a constant which is included in equation (1) to take into account effects such as the liquid reflectance, fibre attenuation, etc.

If the beam was well collimated, the radius of the lens and the reflected beam should be the same. But depending on the alignment tolerances, a small beam divergence can be considered.

As distance increases the collected light at the receiver lens decreases. But, in equal conditions, at a same distance, the collected power increases for lenses having a higher diameter. Different behaviours can be expected depending on the lens parameters. So various sensor heads with different lenses have been developed as reported in section III.

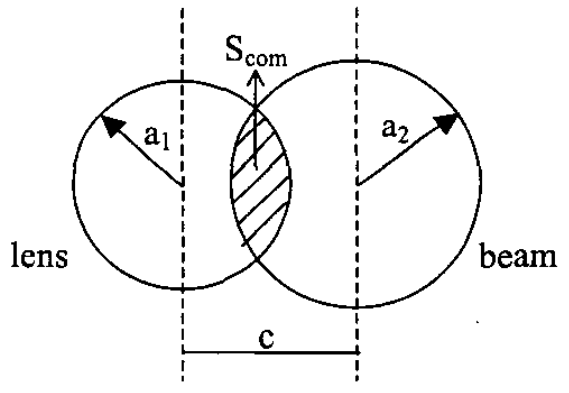

Fig. 3. Common area between the lens and the reflected beam on the liquid surface. 


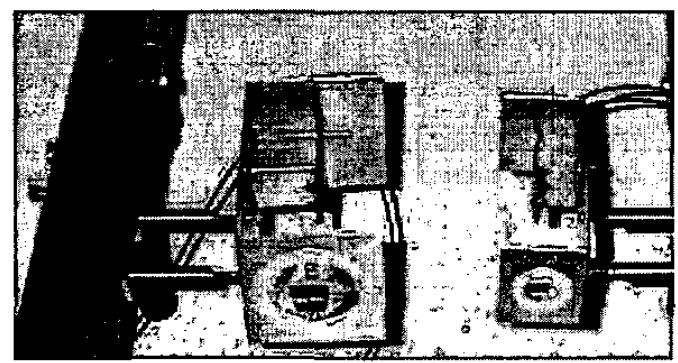

Fig. 4. Photograph of 2 sensor heads.

\section{B. System architecture.}

The light signal used to measure the level is emitted by the generation unit, transmitted to the tank and collected once it is reflected off the liquid surface by the sensor heads. It is converted to the electrical domain by the processing unit to properly extract the desired information and adequate the signals to the microcontroller. These signals are sent to the Personal Computer $P C$, via RS232 port. A software application is developed to configure the system and show the measurement results.

Scalability of the system is reached using Frequency Division Multiplexing (FDM) technique for addressing the signals of the multiple sensors. Each sensor head uses a laser diode modulated at a specific frequency, after being reflected at the liquid surface they are optically multiplexed in a single fibre and transmitted to the processing unit.

1) Sensor heads: They are made of the emitter and receiver fibres. Both are POF with a $1 \mathrm{~mm}$ diameter and a 0.47 numerical aperture. Having an attenuation of $0.24 \mathrm{~dB} / \mathrm{m}$ at $650 \mathrm{~nm}$. They also include the collimating/focusing lens and the mechanical parts to align the sensors perpendicular to the liquid surface. In our design we have used a fibre receptacle base, which is supported by a base surface in " $L$ " shape where the lens is placed in front of the fibres [10]. The lens determines the range and sensitivity of each sensor. The photograph of two sensors heads are shown in Fig. 4. These sensors have lenses with different focal length and diameter and are made of different materials.

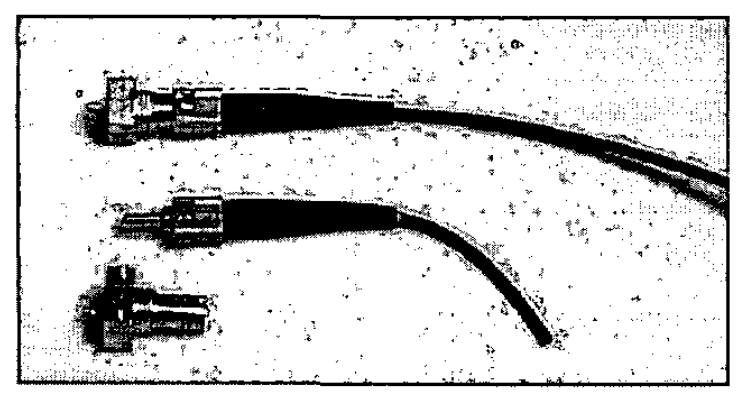

Fig. 5. Photograph an ST fibre pigtail and the laser housed in a ST connector.
2) Generation, detection and processing unit (GDP unit): Generation circuits include the laser diodes, S6505MG ( $5 \mathrm{~mW}$ maximum output power), that we have housed in metal ST-connectors (see Fig. 5) using the thermal bonding compound TBS from Electrolube. This compound has an excellent tensile strength, a very good thermal conductivity and excellent electric insulation characteristics. The generation circuits have also the laser diode drivers IC-WJ from IC haus in analog modulation during $\mathrm{CW}$ operation, in the $\mathrm{kHz}$ range, with stabilisation of the average power. These drivers are excited using a differential pair with discrete bipolar transistors BC547 and an XR-22606 function generator, from Exar Integrated System, operating as a sinusoidal generator.

A regulator with a current limit and a slow turning on circuit is implemented to prevent the laser diode destruction. MOSFET Digital switches (BUK556-60) are used to control the driver activation.

The detection includes the reception and the demodulation circuits. The first one has the optical to electrical (OE) converter, using a photodiode IF D91 from Industrial Fiber Optics housed in a "connector-less" style POF package with a $0.2 \mu \mathrm{A} / \mu \mathrm{W}$ responsivity @ $632 \mathrm{~nm}$ and a rise and fall times of 5 ns. A transimpedance amplifier follows it, made of an operational amplifier LF357 with a capacitance in the feedback loop to filter the noise at high frequencies. Amplification and filtering is reached using 3 inverting stages with integrated circuits TL081 and a cw decoupling capacitor. The last stage has a variable gain to optimise the final gain.

The demodulation circuit is the responsible to extract the information of each sensor. A block diagram showing the different elements can be seen in Fig. 6. An 8-channel multiplexor DG528 from MAXIM has been used. DC decoupling is accomplished using TL081 in an inverting configuration with a capacitor at the input. AD633 multiplier from Analog Devices, a second order Sallen-Key low-pass filter and an inverting amplifier, both with TL081OA, are used.

A logarithmic stage allows having a linear behaviour of the calibration curve, because there is an almost exponential dependence from the measured output power and the liquid

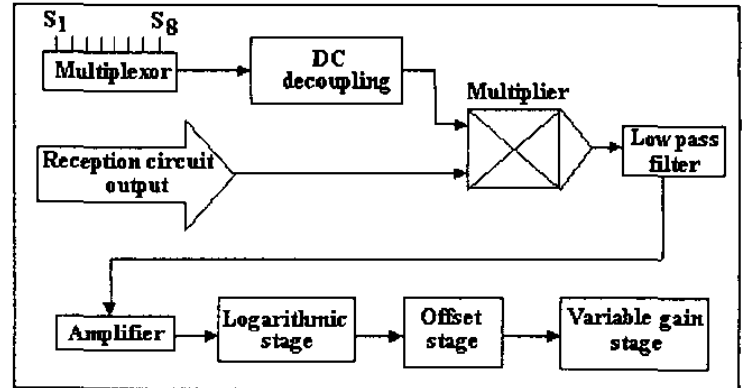

Fig. 6. Block diagram of the demodulator circuit 


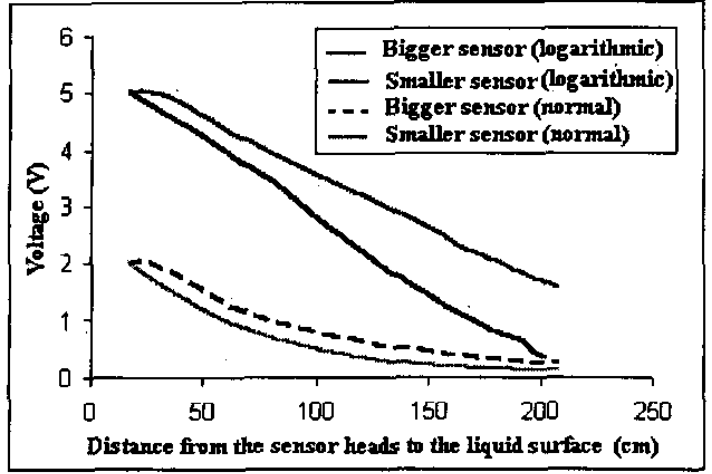

Fig. 7. Calibration curve of the 2 sensors heads before and after the logarithmic stage.

level in the tank (see Fig. 7). This linear dependence allows having the same precision in the whole range. The offset stage is used to adjust the output power at $0 \mathrm{~V}$ when the tank is empty or when the liquid level in the tank is at the minimum position. It is made of a resistive divider connected to a voltage regulator to avoid undesirable voltage instabilities and a non-inverting amplifier. A jumper is used to allow a connection to 8 different offset circuits in case of multiplexing different sensor heads. A final variable gain stage is used to adjust the output power at $+5 \mathrm{~V}$ when the tank is full.

The processing unit includes the acquisition system that converts the analog signals from the demodulator to the digital input signals to the microcontroller. It is used an 8 bits CMOS A/D converter, ADC0804LCN from National Semiconductors, with a reference voltage of $2.5 \mathrm{~V}$ supplied by an LM336. Optionally a variable reference voltage can be used in case of having different sensors as it has been done with the offset circuits. Microcontroller output signals are changed to the voltage levels compatible with the RS232 protocol using the ST232CN chip.

The basic functions of the microcontroller are controlling the system and the communication with the PC. Controlling actions includes:

- Laser diode drivers ON and OFF control.

- To decide the sampling sequence of the different sensors and actuation over the multiplexer control signals.

- The selection of the number of measurements to be taken in each sensor.

The communication with the $\mathrm{PC}$ is full duplex:

- From the PC to the microcontroller for configuring the system.

- From the microcontroller to the PC for sending the measurements.

ATMEL AT89C52 controls the activation of the 8 laser diode drivers through its port 2 . The information of the activation stage of each sensor is saved in a specific register. Addressing of the sensors is done in an ascendant sequence, first it is verified if the sensor is $O N$ and in the affirmative case the measurements are taken. This process is controlled through port 1. The information sent from the PC includes

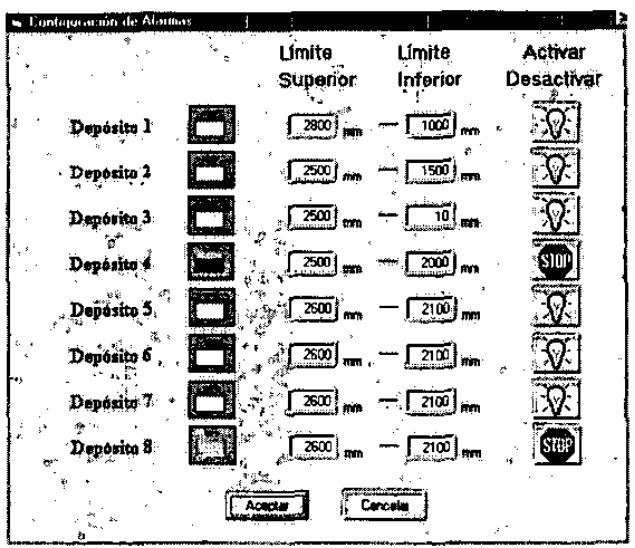

Fig. 8. Software application. Alarms level configuration

the number of sensors, their stage of operation, the number of measurements in each sensor and the interval between measurements.

3) PC \& software application: Data reception, postprocessing and monitoring are done in the PC. A userfriendly software interface is developed in Visual Basic to show, in real time, the level in the different tanks and the alarms activation in case of surpassing predetermined risk levels. Another feature included is the configuration of the system: the number of tanks and sensors in each tank, the type of liquid, the number of samples to be taken in each measure and the interval between measurements.

An example of one of the software application screens, the alarm levels configuration, selected in the tools menu, is shown in Fig. 8. In this case, 8 tanks are defined with an upper (Limite Superior) and lower (Limite Inferior) level of risk, having 6 alarms $\mathrm{ON}$ and 2 OFF.

On Fig. 9 it can be seen the level measurements graphically and numerically in $\mathrm{mm}$, the alarm levels stage and its value if they are ON. In tank (depósito) 6 the risk level is surpassed so an alarm message (ALARMA) appears on the screen.

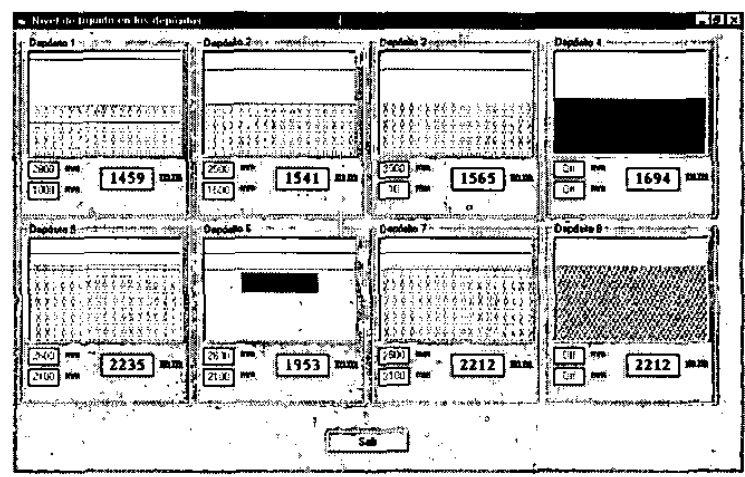

Fig. 9. Software application. Level measurements and alarms stage in each tank. 


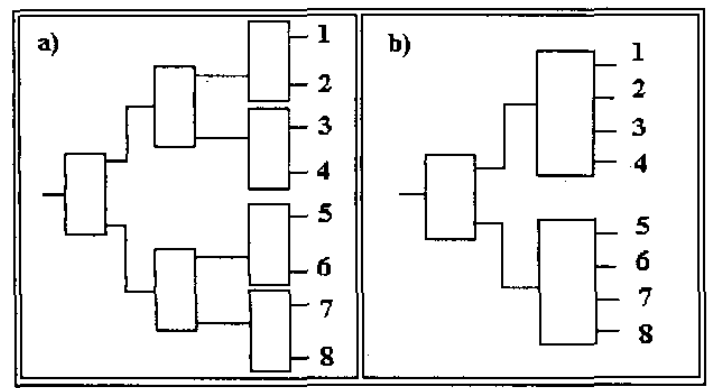

Fig. 10. Optical multiplexing of 8 sensors in a single fibre

This software application can also be used for inventory purposes.

4) Optical multiplexing and transmission: Scalability of the system is achieved using FDM technique. This feature has

conditioned the generation, detection and processing unit design and implementation. But, apart from the electronics and optoelectronics included in the previous subsections, it is necessary to recombine all the light signals in the optical domain for transmitting them to the GDP unit using a single optical fiber, allowing a wider intrinsically safe area in the measurement process. To do so optical recombiners are used. In Fig. 10, two configurations are shown. The first one, Fig. 10.a., uses $2 \times 1$ devices in cascade to multiplex all the sensor heads. The other proposed option uses $2 \times 1$ and $4 \times 1$ devices. Also $8 \times 1$ devices can be used but they have high losses and are less commonly fabricated.

The output single fiber is long enough to connect the whole sensor heads with the GDP unit. In typical petrol stations, the link will be of tenths of meters.

\section{TESTS}

In the following we are going to describe the most relevant tests we have done to the different blocks of the system.

Laser diode drivers operation has been tested. To do so, apart from measuring its bandwidth, it has been measured the average and peak to peak output power during 12 hours using a set up automatically controlled through HPVEE. The results show a $1.3 \%$ deviation in the average power and a maximum $3 \%$ deviation in the peak to peak power during the 12 hours test period. It has been measured that losses in the laser diode output for being embedded in metal ST connectors are of $1.7 \mathrm{~dB}$.

Software application has been validated. Different screens can be seen under operation conditions in Fig. 8 and Fig. 9.

Two different sensor heads (shown in Fig. 4) have been calibrated using our multi-sensor system. To do so, we have adjusted both sensors. The minimum output of the system in the small sensor at $207 \mathrm{~cm}$ is set to $0.5 \mathrm{~V}$ with the offset circuit. The maximum output of the small sensor is set to $5 \mathrm{~V}$ modifying the gain stage for a $17 \mathrm{~cm}$ distance. The measured

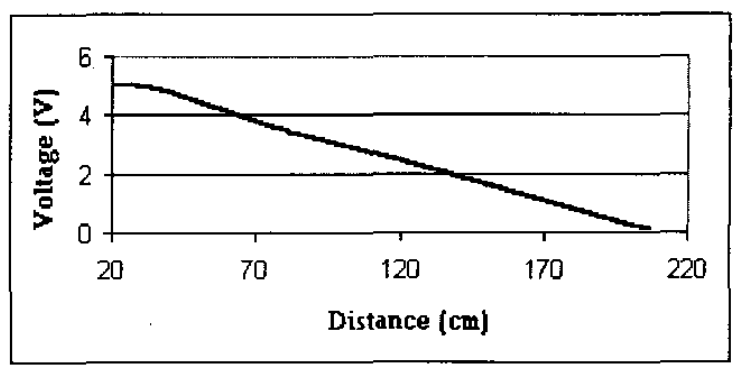

Fig. 11. Full calibration for the bigger sensor.

calibration curves can be seen in Fig. 7. We see that the logarithmic stage effectively produces a linear output response. On the other hand, as it was expected, the bigger sensor (having a bigger lens diameter) has a smaller slope, because can collect much more light at the same distance (see Fig. 3) and the output voltage range was adjusted for the other sensor head. So if the potentiometer is changed for adjusting the $5 \mathrm{~V}$ output for the $2 \mathrm{~m}$ range in this sensor it will have a higher sensitivity.

A full calibration is carried out for the bigger sensor, including a greater number of measuring points than in the calibration reported in Fig. 7. Measurements are taken each $1 \mathrm{~cm}$. The first measure is taken when the liquid surface is at $207 \mathrm{~cm}$ from the sensor head. At this point the offset is set to have $0 \mathrm{~V}$ at the output. The last measurement is taken at $17 \mathrm{~cm}$, setting the gain to have a $5 \mathrm{~V}$ output signal. The measures have been averaged in the oscilloscope TDS210, having a greater number of samples in the longer distances. Measured calibration curve is shown in Fig. 11 and it can been seen that the system has a good linearity. A straight line has been fitted to the measured output voltage over the span $37 \mathrm{~cm}$ to $207 \mathrm{~cm}$. System linearity is calculated as the maximum deviation between actual and linear predicted output voltage. Linearity is better than $1.5 \%$ FS from $37 \mathrm{~cm}$ to $207 \mathrm{~cm}$. The first measurements do not follow the same linear relation, this effect at shorter distances is not as clearly shown in the smaller sensor (see Fig. 7). For that span a system sensitivity of $0.02 \mathrm{~V} / \mathrm{cm}$ is obtained.

Test bench limitations have conditioned the 2 meter range. The output power at the maximum distance measured is high enough to allow increasing the measuring range.

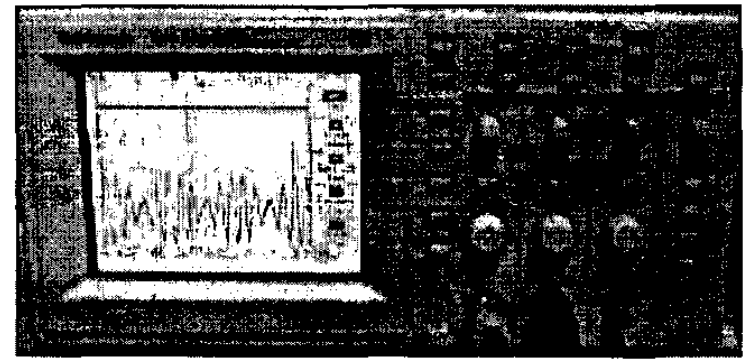

Fig. 12. Photograph of the measurements in the oscilloscope. Detection unit (above), and reception circuit (below) outputs. 
System resolution is limited by AD conversion so it can be improved by employing a better conditioning. In the actual design with an 8 bit $A D$ converter for the $2 \mathrm{~m}$ range a resolution $<0.5 \% \mathrm{FS}$ is obtained.

Optical multiplexing of 2 sensors heads have been carried out, using a $2 \times 1$ POF splitter from Ratioplast with $4 \mathrm{~dB}$ insertion losses and following the configuration shown in Fig.10.a. Metal connectors are used to connect receiver fibre pigtails and POF splitter input. The output signal detected by the OE converter before being demodulated, can be seen in Fig. 12. It is a photograph of the TD210 oscilloscope screen. In the inferior trace it is shown the sum of 2 modulated signals coming from the 2 sensor heads, each one is addressed at a different frequency. The signal of each sensor is demodulated using the circuit of Fig. 6, and a continuous voltage signal is obtained. One of the demodulated signals can be seen in the upper trace of Fig. 12.

As a summary of the device behaviour, system specifications are reported in Table I.

TABLE I

PROTOTYPE SPECIFICATIONS AND PARAMETERS

\begin{tabular}{|l|l|}
\hline power supply & $\pm 12 \mathrm{~V}$ \\
\hline laser diode output power & $5 \mathrm{~mW}$ \\
\hline laser diode wavelength & $650 \mathrm{~nm}$ \\
\hline connector /fiber & ST/POF 1mm diameter, \\
& $0.47 \mathrm{NA}$ \\
\hline Serial communication & 10 bits: 1 start bit, 1 stop bit, \\
& 8 bit data, 9600 bauds \\
\hline Graphic monitorization & Visual basic application, PC \\
\hline range & $>2 \mathrm{~m}^{(1)}$ \\
\hline linearity & Better than I.5\% FS \\
\hline resolution & $<0.5 \% \mathrm{FS}^{(2)}$ \\
\hline
\end{tabular}

(1) due to limitations in the test bench

${ }^{(2)}$ can be improved employing a 10 bit $\mathrm{AD}$ converter.

\section{CONCLUSIONS}

A scalable system, intrinsically safe is developed, to allow measuring the level in different tanks in flammable atmospheres, multiplexing the signals and transmitting them to the PC located in the central office. A user-friendly interface is developed to show in real time the level in the different tanks and alarms activation in case of surpassed predetermined levels. This software application can be also used for inventory purposes and to initially configure the system. The multi-sensor system has been tested using 2 different sensor heads using a $2 \times 1$ optical splitter/combiner and connectors for optically multiplexing the output signals. A full calibration is carried out for one of the sensors and a good linearity better than $1.5 \% \mathrm{FS}$ is obtained in a $2 \mathrm{~m}$ range. This range is limited by the present test bench. Resolution better than $0.5 \%$ FS is obtained and can be improved employing a better conditioning.

\section{ACKNOWLEDGMENT}

The authors gratefully acknowledge the contributions of J.E.Cerdá and J. Pérez in the preliminary prototype. This work was supported by Comunidad Autónoma de Madrid (CAM-07T-0011-2001).

\section{REFERENCES}

[1] Informe medioambiental de 1998 de Repsol S.A. Validado por Dames\&Moore.

\section{[2] http://www.edomexico.gob.mx/se/proyvapores.htm}

[3] K. Nakahira, T. Kodama. S. Morita and S. Okuma, "Distance measurement by an ultrasonic system based on a digital polarity correlator". IEEE Transactions on Instrumentation and Measurement, Vol 5, $\mathrm{n}^{\circ} .6$. December 2001, pp.1748-1752.

[4] "Laser liquid level gauge with diffuser", US5648844, 15.07.97.

[5] Iwamoto K. y Kawata I. "Liquid-level sensor with optical fiber", App. Optics, Vol.31, n¹, 1992, 51-54.

[6] L. Yuan, "Automatic-compensated two-dimensional fiber-optic sensor", Optical Fiber Technology, 4, 1998, pp. 490-498.

[7] G. Betta, A. Pietrosanto y A. Scaglione. "A Gray-Code based fiber optic liquid level sensor", IEEE Transactions on Instrumentation and Measurement, Vol.27, n¹, 1998, pp. 174-178.

[8] R. Felgenhauer et al "Fiber Optic displacement sensor with POF", Proceedings of the 1993 Plastic Optical Fibre Conference POF'93, 1993, pp. 171-173.

[9] W099/56093 "Remote sensor head for laser measurements devices", 29-03-1998.

[10] P200002032 "Sistema sensor óptico para medida de nivel de líquidos en entornos críticos", 09-08-2000. 